\title{
Des chemins de traverse
}

\section{Une revue de l'exposition Les villes d'à côté}

Annabelle Fouquet

Université Laval

Zxposition virtuelle conçue par Francine Saillant, Frantz Voltaire et Sarah Bourdages-Duclot dans le cadre de la Semaine d'action contre le racisme (SACR) (Montréal, I7-24 mars 202I). En collaboration avec le Centre International de Documentation et d'Information Haïtienne, Caribéenne et Afro-canadienne (CIDIHCA), le Laboratoire de recherche sur les relations interculturelles de l'Université de Montréal (LABRRI) et le Groupe de recherche sur les imaginaires politiques en Amérique latine (GRIPAL).

Le 17 mars 202I, l'exposition Les villes d’à côté était inaugurée en ligne. À travers des extraits de témoignages, 70 dessins et IIo photographies et vidéos produits en grande majorité par des jeunes résidant dans des quartiers marginalisés de Montréal, Port-au-Prince, Rio de Janeiro et de la région parisienne, cette exposition virtuelle invite à placer le curseur de nos regards loin des représentations médiatiques qui ne restituent bien souvent de la vie de ces jeunes que des ombres déformées. Cette exposition s'apparente dès lors à un exercice de mise en lumière et de redressement de perspectives, nous permettant, pour reprendre les termes d'un des participants, d'aller à rebours des esprits qui «pensent de travers »'.

La proposition initiale est ambitieuse: rapprocher à travers des témoignages, des dessins, des photographies et des vidéos l'expérience de vie de plusieurs jeunes personnes (I8-30 ans) issues des zones marginalisées de quatre métropoles. Au Canada, le projet se déroule au Nord de Montréal, dans le quartier SaintMichel et dans l'arrondissement Montréal-Nord, deux quartiers parmi les plus pauvres du pays, tous deux situés dans un environnement fortement marqué par une histoire ouvrière et par une population issue de l'immigration. En France, le projet se déroule dans la commune de Grigny, en plein cœur 
d'un ancien quartier ouvrier située dans la banlieue sud de Paris, également marquée par une histoire d'immigration qui s'est considérablement accélérée dès la seconde moitié des années 1960. En addition à ces deux contextes, le projet élargit ses frontières en se déployant dans la capitale d'Haïti, au cœur de plusieurs quartiers populaires de la capitale de Port-au-Prince et enfin dans les ruelles de Rocinha, la plus grande favela brésilienne, située au sud de Rio de Janeiro. Alors que certains quartiers populaires de Port-au-Prince sont devenus le théâtre d'affrontements quotidiens entre bandes rivales et font l'objet de violentes opérations policières et militaires (Djems 2019), la favela de Rocinha, est traversée par une guerre entre narcotrafiquants qui sévit simultanément à l'accroissement de la corruption et des violences policières (Soares Gonçalves 2006). Malgré plusieurs écarts manifestes séparant chacun de ces quartiers situés aux 4 coins du globe, (le terme "quartier» est lui-même difficilement adoptable pour chacun des lieux), de nombreux aspects contextuels créent néanmoins de riches espaces de résonnance qui ont probablement stimulé l'intuition des commissaires à l'origine de ce projet.

\section{Une exposition polyphonique}

Initialement pensée afin d'être présentée "physiquement», cette exposition devait à l'origine permettre de relier entre eux les dessins produits par les participants, quelles que soient leur provenance, grâce à une petite césure découpée au centre des feuilles de papier. Ce dispositif de reliure aurait ainsi permis de matérialiser la mise en relation de ces quatre contextes. En raison de la pandémie, cette exposition "physique» n'a finalement pas pu avoir lieu. La mise en relation de l'ensemble de ces matériaux s'opère donc de manière virtuelle, au fil des "galeries» accessibles sur la plateforme virtuelle de l'exposition. Dans la continuité d'initiatives ethnographiques visant à expérimenter de nouvelles formes de méthodologies collaboratives et créatives (Culhane 2017), cette exposition virtuelle nous propose une navigation à travers les espaces de vie quotidiens, intimes et imaginaires de l'ensemble des participants du projet. En poussant plus loin le souhait d'une «anthropologie non autoritaire ", "relationnelle» "dialogique » et «multilocalisée», déjà énoncé dans le Manifeste de Lausanne (Saillant, Mondher et Graezer Bideau 20II, I5), l'approche méthodologique de ce projet de recherche et de création ainsi que les choix éditoriaux de la plateforme virtuelle permettent aux visiteurs de reconstruire leurs propres récits sur la base d'une matière polyphonique et polymorphe divisée en quatre volets: I) «ambiances urbaines»; 2) «discriminations et préjugés»;3) «droit à la beauté et 
fierté»; 4) «agir, transformer, espérer, imaginer». Dans l'ensemble de ces volets, alors que les dessins et les clichés photographiques des jeunes participants s'entremêlent indépendamment des contextes locaux, en donnant à voir une continuité souvent troublante entre leurs expériences de vie, leurs paroles (très justement fragmentaires et économes) sont de leur côté divisées selon les quatre contextes géographiques. Cette balance entre une mise en liaison et un rappel des contextes locaux se trouve suffisamment équilibrée pour que l'exercice réflexif s'opère bien du côté du visiteur, le poussant à réfléchir et à sentir ce qui traverse communément ou distinctement l'ensemble des expériences de vie rassemblées par le projet.

\section{Des espaces urbains fragmentés}

Une des premières résonnances qui a marqué ma visite virtuelle est le constat d'une fragmentation de l'espace métropolitain au sein des quatre contextes nationaux. Face à une absence d'intégration et de planification urbaine, cette exposition virtuelle prend à contre-pied la fracture spatiale, économique et symbolique qui semble séparer ces quartiers périphériques des grands centres urbains. Elle donne place aux paroles et aux regards critiques de jeunes générations défavorisées en termes d'accès aux services publics, au marché du travail et aux infrastructures culturelles et éducatives.

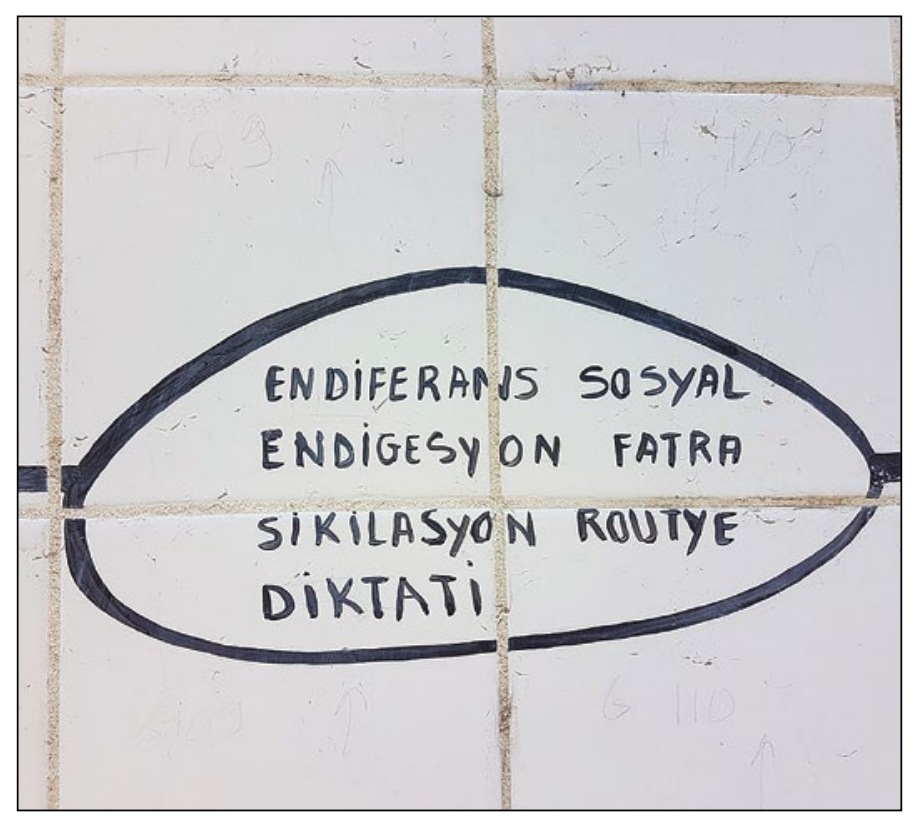

Figure 1. «Indifférence sociale, indigestion de déchets, circulation routière, dictature » (Équipe Haïti). 
Comme cela transparaît au fil de certaines images dessinées ou photographiées par les jeunes de ces différents quartiers, l'expérience de violences racistes sur la race s'intègre ici à l'expérience plus large d'une forme de ségrégation tout autant territoriale, économique que symbolique. Comme le précisent les commissaires de l'exposition, «le racisme fait partie des discriminations vécues [...] mais il se situe comme un phénomène à maîtriser par une lutte globale contre des préjugés tenaces accolés au regard déformé d'un groupe sur une collectivité dépeinte défavorablement. ».

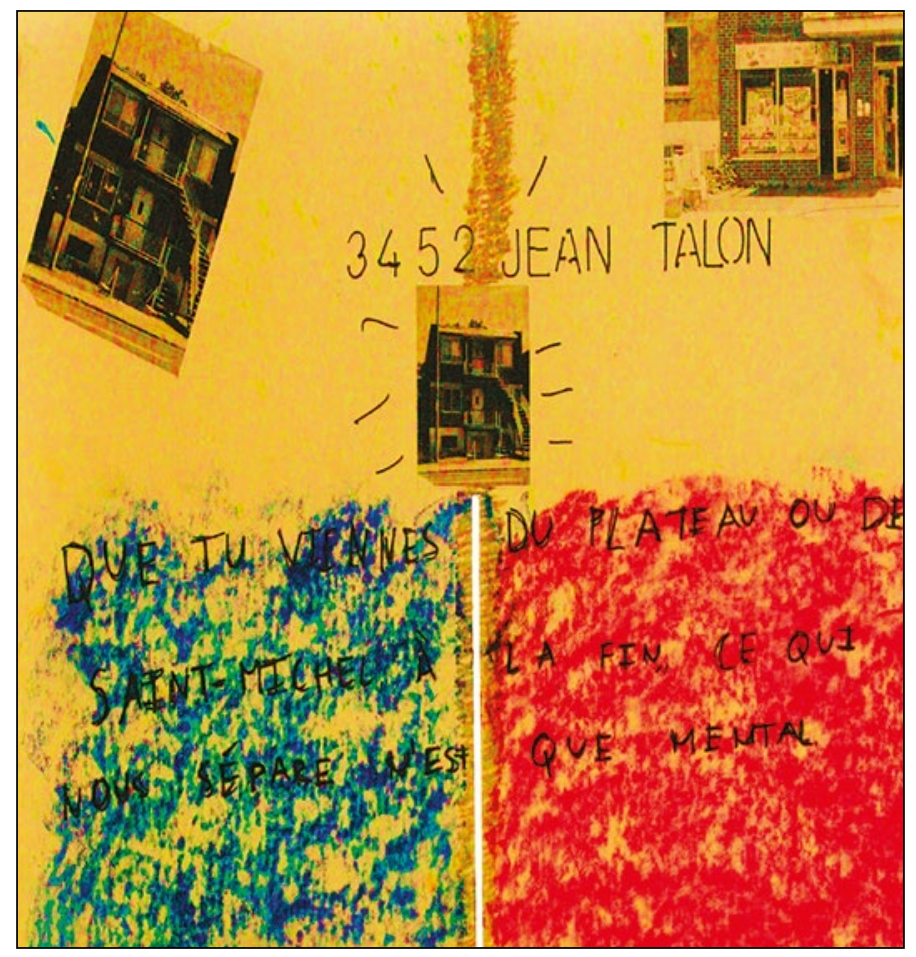

Figure 2. «Que tu viennes du Plateau ou de St-Michel, ce qui nous sépare n'est que mental » (Équipe Montréal). Nombreux jeunes ont interprété les petites «césures » au centre des feuilles de papier afin de questionner avec créativité les frontières spatiales, économiques et symboliques qui leur sont imposées.

\section{Le droit à la normalité et à la beauté}

Si certains des dessins, des photographies ou des paroles de participants témoignent bien de la dureté des formes de violence imposées aux jeunes participants de ce projet, ce qui frappe de prime abord lorsque l'on navigue à travers les galeries de l'exposition, c'est l'omniprésence d'une relation complexe et non dichotomique entre expérience de la violence et normalité, stigmatisation et fierté, marginalisation et engagement citoyen, oubli et souhait accru de visibilité, désir d'ailleurs et appartenance au quartier. 


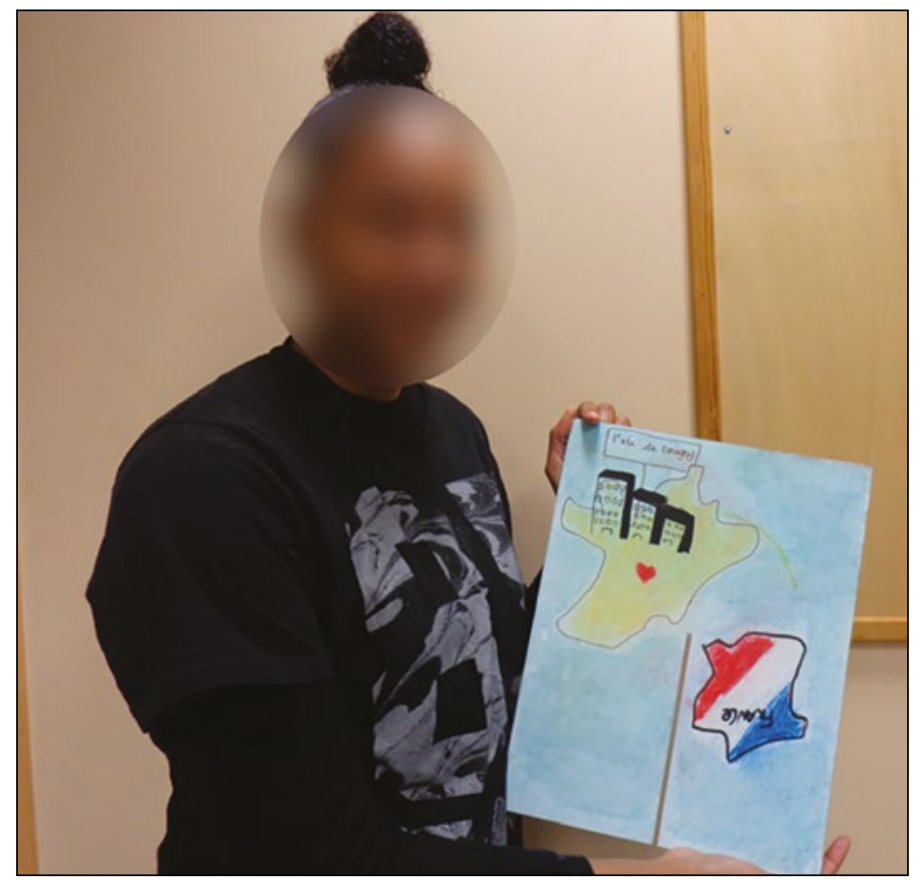

Figure 3. «|'habite une île à côté d'un pays » (Kadiatou). France, Grigny. Crédit photographique: Francine Saillant.

Comme cela a déjà été évoqué, il peut être parfois plus facile de s'identifier à une ville et à un «espace de vie immédiat » comme un quartier, qu'à une nation (White, Lomamba et Hsab 20I7, II), notamment lorsque la confiance envers les services publics et forces de l'ordre nationales est rompue. Un premier axe de continuité qui émane des quatre axes thématiques choisis réside peut-être dans cette sensation d'appartenance commune au quartier, évoquée à travers les représentations photographiques et les dessins de lieux et d'objets aimés: des maisons colorées et fresques murales de Rocinha aux tap-taps de Port-au-Prince; des étals de marché aux produits offerts dans une épicerie de quartier; des parcs et oasis urbains à la simplicité d'un banc ou d'une ruelle... La beauté des choses simples et la familiarité des lieux fréquentés que nous donnent à voir les images exposées s'apparentent à des stratégies efficaces afin d'inverser les regards stigmatisant et clivants qui sont généralement imposés à ces lieux et leurs habitants par ceux qui ne les habitent pas. Comme les commissaires le rappellent justement, montrer le «beau » ici, ça n'est pas pour le magnifier mais pour nous rappeler tout simplement, que le «le beau existe aussi chez soi et que cela doit être vu.», 


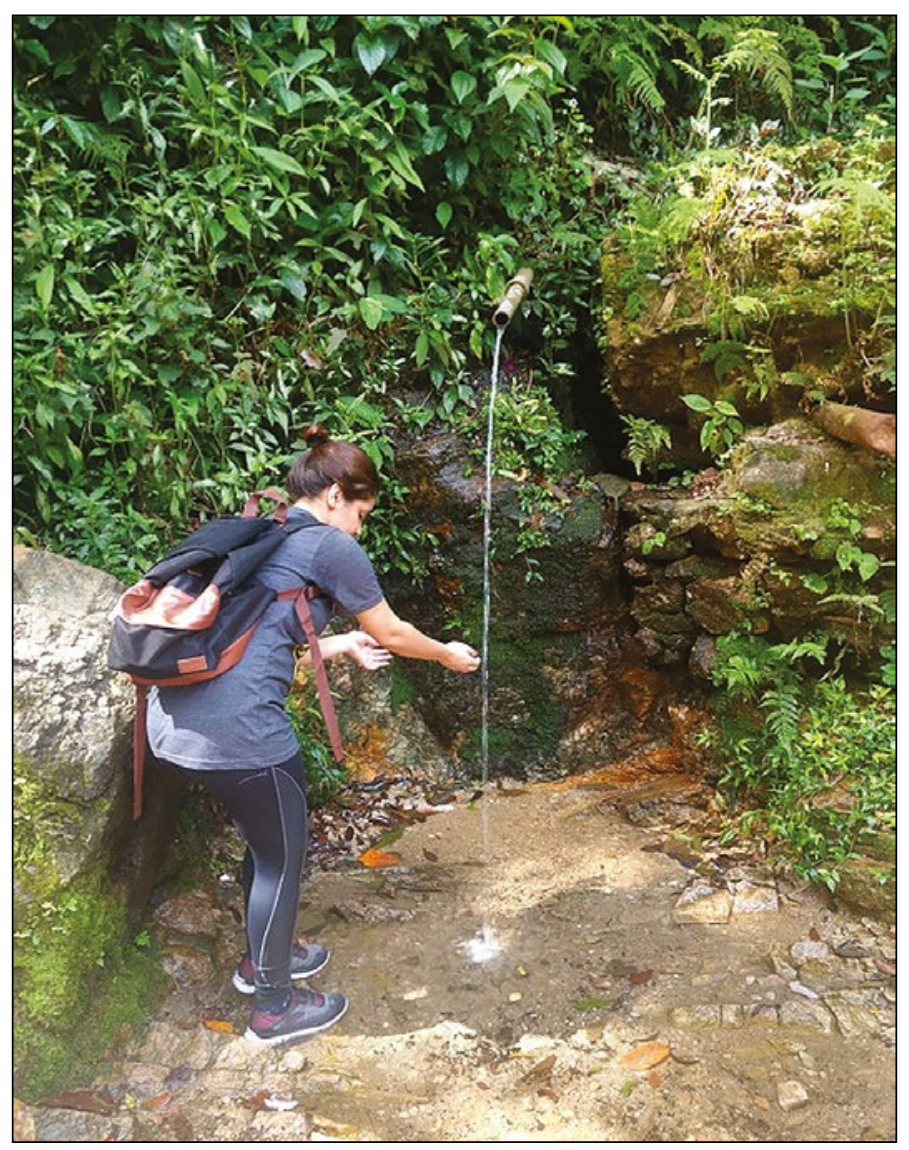

Figure 4. «La source d'eau potable au sommet de Rocinha 》(Équipe Brésil).

La centralité cohésive des associations et des organismes communautaires au cœur de ces différents quartiers (cirque, ciné-club, centre de la vie sociale, groupe de théâtre... $)^{2}$ est aussi au cœur de cette sensation de «chez soi » et d'attachement au lieu et à la collectivité, qui ressort des regards et paroles de ces jeunes. Elle nous rappelle de quelles manières l'appartenance et l'appropriation d'un lieu se manifestent aussi à travers l'engagement et l'implication dans celui-ci (Gagné 2005, 59). À travers les nombreuses mentions d' «union », «d'entraide» et d'exemples d'initiatives collectives qui transparaissent dans les paroles et les images de ces jeunes, ce projet de recherche et de création s'inscrit de manière originale dans la continuité des approches anthropologiques de la citoyenneté qui nous donnent à voir une citoyenneté «horizontale (Neveu 2009, 27), construite à travers les réseaux de solidarité tissés entre les citoyens de ces quartiers. Elle nous donne aussi à voir une dimension non plus seulement sociale, mais profondément intime du droit, alors que celui-ci se retrouve mis en image et en imagination à travers les nombreuses revendications qui émanent des œuvres et paroles des participants du projet. L'ensemble de cette matière 
recueillie nous présente finalement l'envers, ou, pour reprendre les termes des commissaires, «l'inversion» des représentations médiatiques d'une jeunesse jugée en périphérie de la citoyenneté et du droit.

\section{«Je connais le monde entier à travers mon quartier» (Montréal, Canada)}

La diversité au cœur de ces quartiers apparaît également comme une source d'appartenance et de fierté, plus particulièrement évoquée chez les jeunes de Montréal et Grigny. L'apprentissage de différences linguistiques et culturelles semble, pour certains, se transformer en une plus-value et en un répertoire de connaissances dans lequel puiser. Un répertoire auquel s'ajoute une autre forme de savoir, plus politique, induit par l'expérience de l'inégalité. Source de combativité, la «lutte» contre les inégalités apparaît effectivement pour plusieurs d'entre eux comme un «levier» permettant de «se surpasser» et d'aller encore "plus loin», en faisant de ces jeunes des sortes de «guerriers» contemporains aux forces décuplées. Dans certains cas, aller «plus loin » signifie traverser de l'«autre côté» de la rue, comme le mentionne explicitement une des participantes du projet, afin de «vivre comme tout le monde», ou encore de façon plus essentielle, afin de réussir à «vivre » et ne plus seulement «survivre ». Pour d'autres, davantage que de briser une frontière spatiale et économique, «traverser» c'est abolir une frontière symbolique en montrant «qui» l'on est réellement et en prouvant de quoi l'on est capable. Si plusieurs de ces jeunes aspirent à passer "de l'autre côté», cette exposition questionne implicitement le sens dans lequel cette traverse devrait se faire. Dans la continuité des travaux de Saillant sur la reconnaissance et la construction sociale de la différence (Saillant 2004; Saillant, Lévy et Ramirez-Villagra 2017), elle invite les visiteurs à traverser à leur tour une frontière, en reconnaissant la normalité et la dignité de cette jeunesse. En trame de fond de cette déconstruction/reconstruction émerge un questionnement: quel mouvement collectif plus large permettrait de ne plus avoir à traverser de frontières spatiales, économiques ou symboliques? Si ce questionnement invite à des pistes de réponses diversifiées en fonction des contextes, dans le cas français, il nous replonge dans les débats théoriques ayant émergé dès la fin des années 1980, alors que les «quartiers » incarnaient pour certains le passage du paradigme de l'exploitation économique des populations ouvrières urbaines, à celui de l'exclusion spatiale des populations issues de l'immigration (Penven 20I0; Tissot 2007). Si ce paradigme binaire a rapidement été nuancé et complexifié (Wieviorka 1996), il a néanmoins instauré un amalgame 
entre espace et habitants, menant aujourd'hui à un profilage territorial persistant. De cet amalgame ont émergé nombre de politiques urbaines qui n'adressèrent souvent qu'insuffisamment les causes structurelles des difficultés vécues par les résidents de ces quartiers, en ciblant davantage sur leurs responsabilités et capacités individuelles (Bacqué et Biewener 2015), tout en occultant simultanément le processus de «ghettoïsation» des grands centres urbains et des quartiers aisés avoisinants.

En miroir à cette incursion proposée dans l'intimité d'une jeunesse située aux quatre coins du globe, entre l'hémisphère nord et l'hémisphère sud, il aurait pu être enrichissant pour les visiteurs de l'exposition que la plateforme de l'exposition propose une mise en contexte historique et sociale spécifique pour chacun des quatre territoires, et que celle-ci nous informe des réponses politiques (ou de leur inexistence) qui sont jusqu'à présent adressées à cette jeunesse. À ce stade, l'exposition Les Villes d’à côté choisit de s'en tenir essentiellement aux points de vue de jeunes habitants de ces quartiers, bien souvent passés sous silence. Elle choisit également, à juste titre, de valoriser et de visibiliser les organismes et associations de terrains, qui demeurent, face aux politiques publiques, et parfois envers celles-ci, les «instances éducatives informelles» les plus efficaces afin d'ouvrir des trajectoires de vie alternatives, à l'encontre des déterminants sociaux (Penven 20I0).

\section{Une méthodologie créative}

Au-delà de l'aspect colossal de rassembler des expériences de vécu ancrées dans quatre contextes, la force du projet réside dans la mise en œuvre et l'exploration de formes ethnographiques novatrices. À travers les propositions ethnographiques au cœur de ce projet, on peut par exemple souligner la richesse des ateliers de dessin et de cartographie participative mais aussi la pertinence des «entrevues marchées» entremêlées aux prises de vue photographiques, qui ont permis de recueillir une compréhension plus fine de l'expérience de vie de ces jeunes. Au total, entre 2017 et 2018, 30 entretiens individuels, 13 entretiens de groupe (dont de nombreuses «entrevues marchées») et huit ateliers de dessins ont pu être combinés à travers les quatre contextes géographiques. Autant l'exercice de conceptualisation de cette exposition virtuelle que l'ensemble de ces propositions méthodologiques confèrent au projet Les villes d’à côté une dimension résolument pédagogique pour qui s'intéresserait à repenser les frontières de la discipline anthropologique. Cette exposition virtuelle apparaît comme une ressource 
intéressante à mobiliser pour les professeures et professeurs ou les chercheures et chercheurs en anthropologue qui souhaiteraient explorer et faire dialoguer les différentes formes de méthodes ethnographiques.

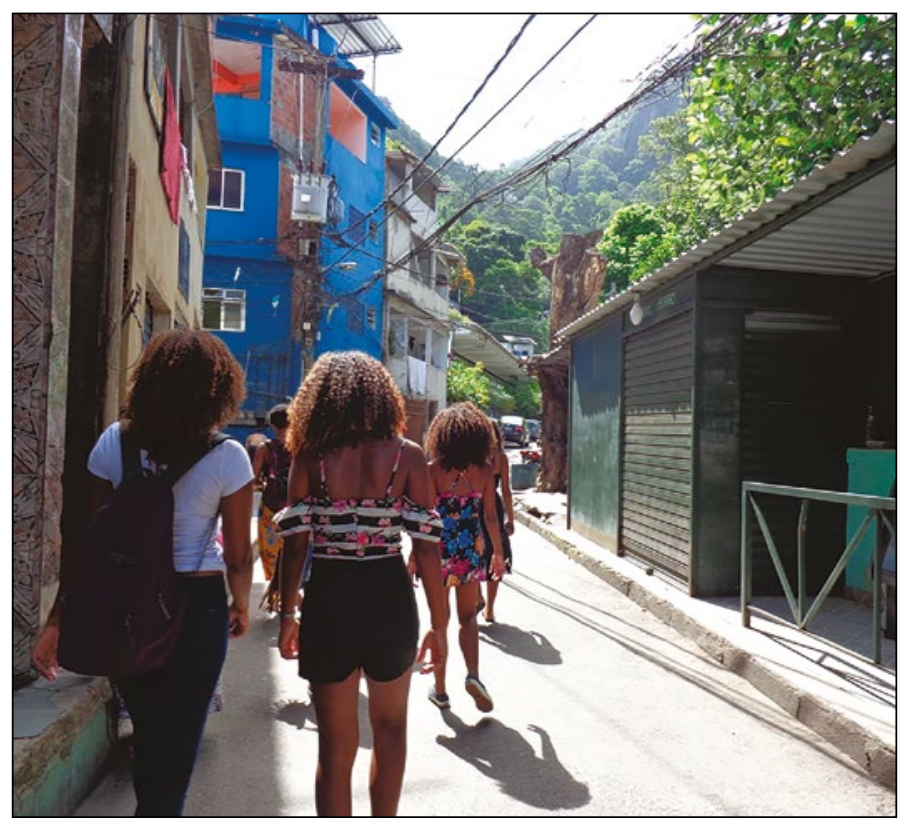

Figure 5. Entrevue marchée. Rocinha, Brésil.

Crédit photographique: équipe de recherche.

Dans le cadre de ce projet, les entrevues marchées semblent avoir permis de relier différentes couches d'expérience du réel, alors que les jeunes marcheuses et marcheurs ont pu à la fois témoigner de leur appartenance aux lieux tout en évoquant ce qui pourrait idéalement advenir de ceux-là. Pour un projet visant à questionner de manière critique la déformation des représentations de la jeunesse dans l'espace public et médiatique, l'entrevue marchée nous rappelle que l'espace public permet encore d'être vu et de proclamer sa place dans la société, tout en dérangeant les idées et relations sociales existantes (Moretti 20I7, 94) ${ }^{3}$. Néanmoins, l'expérience de terrain des chercheurs nous renvoie également au constat que cet espace public n'est pas uniformément accessible selon les contextes. Ces différences semblent, par ailleurs, avoir structuré l'expérience de terrain des commissaires de l'exposition, les amenant par exemple à annuler les marches collectives prévues dans les rues de Port-au-Prince, suite au meurtre d'un jeune homme proche des participants du projet, tandis qu'à Rocinha, ces marches se faisaient bien souvent avec des tirs de balles en trame sonore. L'entrevue marchée apparaît d'autant plus comme un outil pertinent afin de faire l'expérience sensible et incarnée de la fragilité, 
voire de l'impossibilité de notre présence et/ou celles de nos interlocuteurs dans l'espace public. En complément ou en remplacement de celles-ci, les commissaires du projet proposaient des ateliers de cartographie participative, prenant la forme d'ateliers où les participants étaient invités à dessiner leur quartier, de manière tout autant figurée que symbolique.

Les réseaux virtuels (Whatsapp, Messenger), furent également un médium de communication (et de recherche) privilégié afin de communiquer avec les participants du projet lorsque l'accès aux lieux de rencontres étaient parfois compromis, comme à Rocinha et Port-au-Prince, mais également à Grigny, alors que la situation des gilets jaunes rendait de plus en plus difficile l'accès aux banlieues. C'est par ces réseaux que de nombreuses photographies semblent avoir été transmises aux commissaires. Cette mobilisation de l'image photographique ou de la vidéo sur le terrain s'inscrit à son tour dans une longue continuité de recherches en anthropologie et sociologie visuelles (MacDougall I994; Maresca I996; Grimshaw et Ravetz 2005), et dans la continuité de projets de rechercheaction collaboratives, qui mobilisent la technique Photovoice afin de faire émerger du sens par l'acte photographique. Face au médium photographique, et face aux communications virtuelles de manière plus générale, les commissaires rappellent, par ailleurs, que ces méthodes et stratégies de rencontre ne peuvent pour autant être mobilisés de manière unilatérale selon les contextes: alors qu'à Port-au-Prince certaines photos n'ont pu être produites par les jeunes participants, et qu'à Grigny, la majorité des visages de jeunes filles apparaissent de manière floutée, afin de respecter la volonté d'anonymat de celles-ci face aux craintes d'harcèlement sur les réseaux sociaux.

À côté de ces nombreuses disparités contextuelles, tant vécues au sein des entrevues marchées qu'autour des enjeux reliés à la prise de vue photographique, les 70 dessins présentés sur la plateforme web de l'exposition laissent peut-être entrevoir un ultime espace de vie, quasi inaliénable et communément investi par les participants: celui de l'imagination. Plusieurs initiatives en anthropologie ont déjà mobilisé le pouvoir créateur de l'imagination afin d'explorer le réel, en l'abordant soit comme un «terreau de l'action", une "voie d'évasion» (Appadurai 1996 in Kazubowski-Houston et Magnat 2018) ou encore comme un «moteur de nouveauté » permettant de repenser le monde réel (Robins 2010 in Kazubowski-Houston et Magnat 20I8). À travers les dessins présentés, il nous est ainsi donné la chance de nous immiscer sans intrusion dans une dimension intime et imaginée de l'expérience de vie de ces jeunes. Cette dimension ne possède pas de frontières concrètes, hormis 
celles, plastiques, d'une feuille de papier. Et celles-ci n'ont pas tardé à se voir réappropriées et subverties à leur tour par les jeunes créateurs. Comme mentionné, alors que les commissaires du projet avaient initialement pensé à un dispositif qui joindrait ensemble les dessins en leur centre grâce à une césure, de nombreux jeunes ont réinterprété de manière significative ces ruptures dans l'espace de la feuille. Peut-être à l'image de ce qu'ils font quotidiennement face aux frontières spatiales, économiques et symboliques qui leur sont imposées...

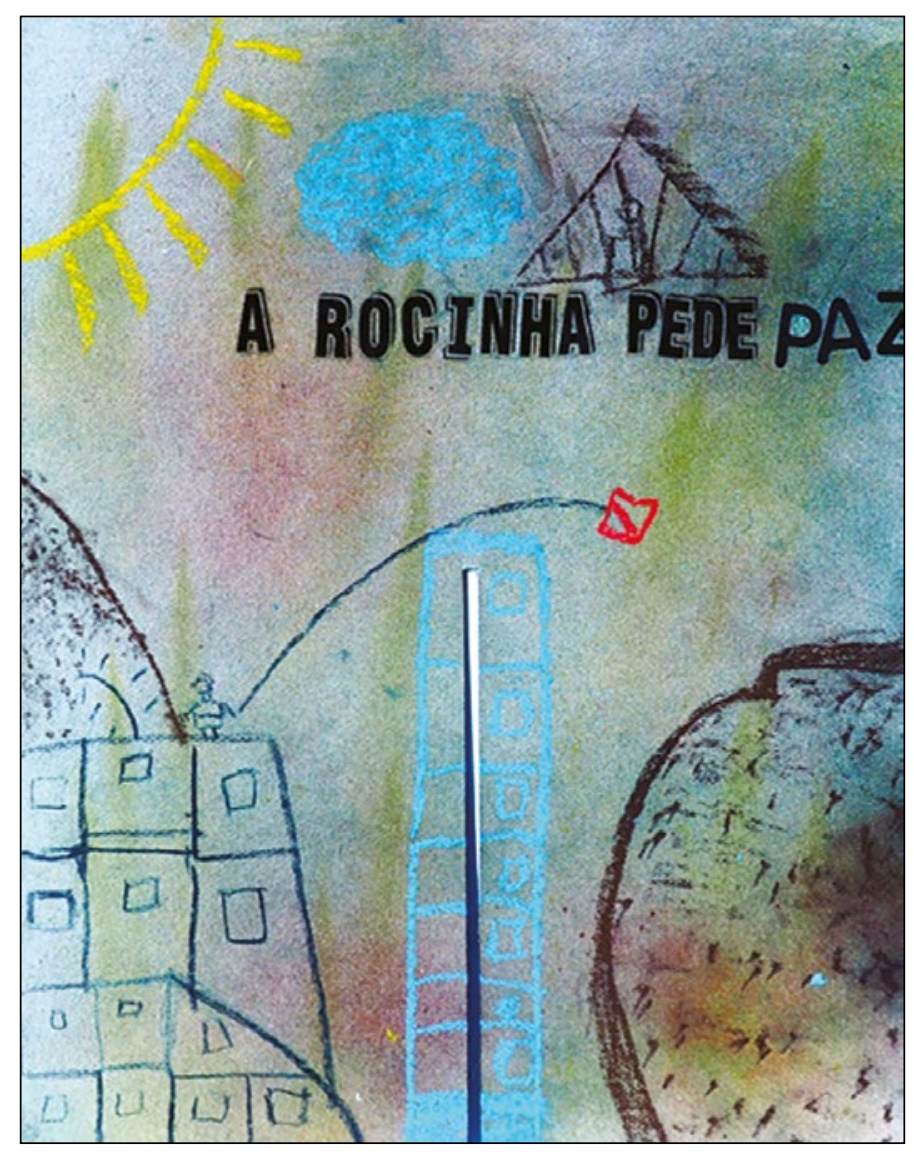

Figure 6. «La Rocinha demande la paix» (Willington).

\section{Une suite?}

De ce vaste projet naîtront probablement un catalogue et un article afin de témoigner du parcours réflexif ayant mené à la plateforme virtuelle de l'exposition. Comme visiteurs, après avoir eu la chance de s'immiscer dans la vie de ces jeunes, on souhaiterait voir ces chemins se poursuivre au gré d'autres rencontres. Les rapprochements d'expériences de vie rendus possibles grâce à la virtualité d'une plateforme virtuelle pourraient-ils à nouveau se reterritorialiser? La création, qui s'apparente ici à une fenêtre de respiration, 
pourrait-elle être à nouveau un vecteur de traverses et de rencontres pour l'ensemble de ces jeunes éparpillés aux quatre coins du monde? Enfin, de quelle manière pourraient être intégrés au projet celles et ceux qui résident "de l'autre côté»? Des échanges et une mise en relation pourraient-ils être également envisagés dans ce sens, afin que d'un renversement de regards, on en arrive potentiellement à une mise en dialogue? Plusieurs questionnements qui appellent inéluctablement à une suite.

\section{Notes}

- Dans cet article, lorsqu'elles ne réfèrent pas à un auteur, les mentions entre guillemets sont directement tirées du site web de l'exposition. Elles proviennent des paroles, des dessins ou des légendes des clichés photographiques des participants.

2 Conçue conjointement au projet de recherche sociale et artistique AJIRA (Action, Jeunesse, Intervention, Réseau, Arts) qui visait à dresser un portrait (non-exhaustif) des organismes et associations de quartier, ce projet d'exposition a largement bénéficié de l'aide de plusieurs organismes afin de mobiliser les jeunes et de rendre possible les activités de création et de recherche.

3 Ce réinvestissement de l'espace public et médiatique se fait également sur le web. À Rocinha, plusieurs jeunes ont lancé sur Youtube leur «Favela Flix». À Montréal, l'initiative web «Occupation Hood», sorte de téléréalité dans laquelle se mettent en scène de nombreux jeunes originaires de Laval, Saint-Léonard et Montréal-Nord, a aussi fait beaucoup de bruit sur les réseaux sociaux. En France, l'école de cinéma Kourtrajmé, est réputé pour avoir propulsé de nombreux cinéastes de la région parisienne, ainsi que plusieurs plateformes de diffusion web initiées par ces jeunes.

\section{Références}

Bacqué, Marie-Hélène, et Carole Biewener, 2015. L'empowerment, une pratique émancipatrice? Paris, La Découverte, collection Politique et sociétés.

Culhane, Dara, 2017 «Imagining: An Introduction ». In Dara Culhane et Denielle Elliott (dir.) A Different Kind of Ethnography, p. I-I2. Toronto, University of Toronto Press.

Djems, Olivier, 2019. «Les territoires de la violence à Port-au-Prince: Entre criminalité et inégalités socio-spatiales». In Thomas Lecomte (dir.) Regards croisés sur la violence. Actes du premier Colloque Annuel du Département d'Anthropologie de l'Université de Montréal. Montréal, Anthro.

Fleury, Cynthia, 2006. Imagination, imaginaire, imaginal. Paris, Presses universitaires de France. 
Gagné, Natacha, 2005. «Être jeune et maori aujourd'hui: L'université comme site de (ré)affirmation et de coexistence ». Recherches Amérindiennes au Québec, 35 (3) : 59-70.

Grimshaw, Anna, et Amanda Ravetz, 2005. Visualizing Anthropology. Portland, Intellect.

Kazubowski-Houston, Magdalena, et Virginie Magnat, 20I8. «Introduction: Ethnographie, Performance et Imagination » Anthropologica, 60 (2): 413-426. https://doi.org/I0.3138/ anth.20I7-00o6-fr.

MacDougall, David, 1994. « Mais, au fait, l'anthropologie visuelle existe-t-elle vraiment?», Cinémaction 64:5I-57.

Maresca, Sylvain, 1996. La Photographie. Un miroir des sciences sociales. Paris, L'Harmattan.

Moretti, Cristina, 20I7. «Walking ». In Dara Culhane and Denielle Elliott (dir.) A Different Kind of Ethnography, p. 9I-III. Toronto, University of Toronto Press.

Neveu, Catherine, 2009. «Comment faire l'anthropologie d'un objet trop lourd? Approche anthropologique de la citoyenneté en France». Anthropologie et Sociétés 33 (2): 25-42. https://doi.org/I0.7202/039296ar.

Penven, Alain, 20Io. Ville et coopération sociale. Paris, L'Harmattan.

Saillant, Francine, Kilani, Mondher et Florence Graezer Bideau, 20II. Manifeste de Lausanne. Pour une anthropologie non hégémonique. Montréal, Liber.

Saillant, Francine, 2004. "Constructivismes, identités flexibles et communautés vulnérables ». In Francine Saillant, Michèle Clément et Charles Gaucher (dir.) Identités, Vulnérabilités, Communautés, p. 19-42. Montréal, Nota Bene.

Saillant, Francine, Joseph J. Lévy et Alfredo, Ramirez-Villagra, 20I7. «Perspectives interculturelles, droits et reconnaissance à Montréal ». Anthropologie et Sociétés, 4I (3) :I55-I79. https://doi.org/I0.7202/IO43046ar.

Soares Gonçalves, Rafael, 2006. «La politique, le droit et les favelas de Rio de Janeiro ». Journal des Anthropologues, I04-I05: I-I7. https://doi.org/I0.4000/jda.4I8.

Tissot, Sylvie, 2007. «Sociologie urbaine et politique de la ville: Retour sur une rencontre autour des 'quartiers d'exil'». In Jean-Yves Authier, Marie-Hélène Bacqué et France Guérin-Pace (dir.) Le quartier, p. 65-74. Paris, La Découverte.

White, W.Bob, Emongo, Lomomba et Gaby Hsab, 20I7. «Présentation: Vers une anthropologie de l'interculturel». Anthropologie et Sociétés, 4I (3): 9-27. https://doi. org/I0.7202/1043040ar.

Wieviorka, Michel, 1996. Une société fragmentée? Le multiculturalisme en débat. Paris, La Découverte. 